\title{
Surrogate-Based Support Vector Machine Method
}

\author{
Yueyang Teng, Yaxin Liu, Xuan Xie, Bin Lu and Yan Kang \\ NO. 3-11, Wenhua Road, Heping District, Shenyang, P. R. China
}

\begin{abstract}
Surrogate-based method (SBM) is used to train a support vector machine (SVM) for discriminating between the elements of two classes of input points. The key idea to develop the algorithm is to replace the minimization of the cost function at each iteration by the minimization of a surrogate function, leading to a guaranteed decrease in the cost function. SBM simultaneously update all of points, which is very different from Platt's sequential minimal optimization (SMO) and Joachims' SVM light. The former handles one point at a time and the latter handles a small number of points at a time. In contrast to the sequential methods, SBM is easy to parallelize. The proposed algorithm has some favorable properties, including the monotonic decrease of the cost function, the self-constraining in the feasible region, and the absence of a predetermined step size and any additional parameter. This paper theoretically proves that the iteration sequence will converge to a sole global solution. Encouraging numerical results are presented on data sets, and SBM provides a performance comparable with that of other commonly used methods as concerns convergence speed and computational cost.
\end{abstract}

Keywords-surrogate; Kuhn-Tucker (KT); conditions; regularization technique; sparsity/smoothness penalty

\section{INTRODUCTION}

Support vector machines (SVMs) are receiving in-creasing attention in recent years, which achieve a linear or nonlinear separating surface to separate points belonging to two given sets. The essence of SVM is a quadratic programming problem, and the basic problem is how to solve the quadratic programming accurately and quickly. The problems have close connection with optimal theory in mathematical programming. SVM is an important example that optimization theory applies in data mining. Before giving a detailed discussion, We now describe our notation. All vectors are column vectors unless otherwise specified. For a matrix or vector, the transpose' will denote the transpose. We denote e as a vector of ones in a real space of arbitrary dimension. The identity matrix of arbitrary dimension is denoted by E. When the two classes of points in the training set is needed to separate by a linear hyper plane, it is natural to use the hyperplane that separates the two groups of points in the training set by the largest margin. Denote the training data set in the n-

dimensional real space $\mathrm{RN}$ by the $\mathrm{M} \times \mathrm{N}$ matrix $\mathrm{A}$, where every row of $A$ represents an point. We classify $M$ points in two classes as specified by a given $\mathrm{M} \times \mathrm{M}$ diagonal matrix $\mathrm{D}$ with $+1 \mathrm{~s}$ or $-1 \mathrm{~s}$ along its diagonal. Denote $\mathrm{w}$ is the normal to the hyperplane $x^{\prime} w=\gamma$ and $\gamma$ determines the location to the origin.

$$
\begin{array}{ll}
\min & \frac{1}{2} \omega^{\prime} \omega+\beta e^{\prime} y \\
\text { s.t. } & \mathrm{D}(\mathrm{A} \omega+\mathrm{e} \gamma)+\mathrm{y} \geq \mathrm{e} \\
& \mathrm{y} \geq 0
\end{array}
$$

where $\beta$ is a penalty parameter and $\mathrm{y}$ represent the nonnegative slack variables because the input points aren't generally linearly separable. The dual to the standard quadratic linear SVM is the following:

$$
\begin{array}{ll}
\min & \frac{1}{2} u^{\prime} D A A^{\prime} D u-e^{\prime} u \\
\text { s.t. } & \beta \mathrm{e} \geq u \geq 0, \quad e^{\prime} D u=0
\end{array}
$$

It can be noted that the original and dual problems are not positive definite in general, which may lead to many solution. Also, there is too many constraints including an equality constraint and bound constraints, which must consume special computational cost to handle such as SMO and SVM light. O. L. Mangasarian et al change the 1-norm of y to a 2-norm squared which makes the constraint $\mathrm{y} \geq 0$ redundant and append the term $\gamma^{2}$ to $\omega^{\prime} \omega$. This leads to a variant strong convex SVM model:

$$
\begin{aligned}
& \min \quad \frac{1}{2}\left(\omega^{\prime} \omega+\gamma^{2}\right)+\beta y^{\prime} y \\
& \text { s.t. } \quad D(\mathrm{~A} \omega+\mathrm{e} \gamma) \geq e
\end{aligned}
$$

The dual of this problem is:

$$
\begin{aligned}
& \min \quad \frac{1}{2} u^{\prime}\left[\frac{E}{\beta}+D\left(A^{\prime} A+e e^{\prime}\right) D\right] u-e^{\prime} u \\
& \text { s.t. } \quad u \geq 0
\end{aligned}
$$

Now we obtain a reformulated SVM with only nonnegative constraints. The dual variables $u$ may directly determine the separating surface $(\omega, \gamma)$, which generate the classification rule sign $\left(x^{\prime} \omega-\gamma\right)$.

$$
\omega=A^{\prime} D u, y=\frac{u}{\beta}, \gamma=-e^{\prime} D u
$$


To develop the nonlinear kernel classifier, For $A \in R^{m \times l}$ and $B \in R^{l \times n}$, denote the kernel $K(A, B)$ maps $R^{m \times n} \times R^{l \times n}$ into $R^{m \times n}$, where the linear kernel and the Gaussian kernel are two typical special cases. With the linear kernel replaced by the nonlinear kernel product, we obtain the same programming problem in mathematical nature with:

$$
\begin{aligned}
\min & \frac{1}{2} u^{\prime}\left[\frac{E}{\beta}+D\left(K\left(A, A^{\prime}\right) K\left(A, A^{\prime}\right)^{\prime}+e e^{\prime}\right) D\right] u \\
& -e^{\prime} u
\end{aligned}
$$

s.t. $u \geq 0$

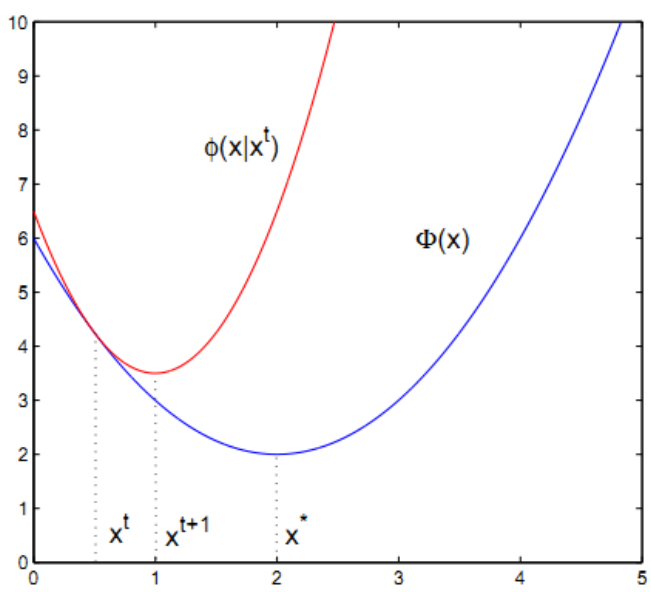

FIGURE I. GRAPHICAL INTERPRETATION OF A SINGLE ITERATION OF THE SURROGATE-BASED ALGORITHM

The surrogate $\varphi\left(x \mid x^{t}\right)$ is bigger than the original function $\phi(X)$ and they are equal at $x=x^{t}$. The surrogate-based algorithm chooses $x^{t+1}$ as new iteration, which minimizes $\varphi\left(x \mid x^{t}\right)$ and guarantees the objective function to decrease in each step.

For an input point $\mathrm{x}, K\left(x^{\prime}, A\right)$ is a row vector , and the linear classifier is replaced by the nonlinear one sign $\left(K\left(x^{\prime}, A\right) D u-\lambda\right)$

\section{MethodOLOGY}

As mentioned in many articles, a surrogate function as defined below is useful in algorithm derivation as well as convergence proof.

Definition 1: (Surrogate function) Denote as a surrogate function for $\phi(X)$ at $x^{t}$ (fixed) if $\varphi\left(x^{t} \mid x^{t}\right)=\phi(X)$ and $\varphi\left(x \mid x^{t}\right) \geq \phi(X)$. It can be concluded that (x) decreases under the update $x^{t+1}=\min _{x} \varphi\left(x \mid x^{t}\right)$ because of

$$
\varphi\left(x^{t+1}\right) \leq \phi\left(x^{t+1} \mid x^{t}\right) \leq \phi\left(x^{t} \mid x^{t}\right)=\varphi\left(x^{t}\right)
$$

Figure 1. shows the diagrammatic sketch of surrogate. There are two simple and important properties for the constructed surrogate functions, which will be useful for the algorithm derivation.

- If $\varphi\left(x \mid x^{t}\right)$ is a surrogate function of $\phi_{\text {mid }}\left(x \mid x^{t}\right)$ at $x^{t}$ and is that of $\phi(X)$ at $x^{t}$, then $\varphi\left(x \mid x^{t}\right)$ is a surrogate function of $\phi(X)$ at $X^{t}$.

- If $\varphi_{1}\left(x \mid x^{t}\right)$ and $\varphi_{2}\left(x \mid x^{t}\right)$ are respectively the surrogate functions of $\phi_{1}(x)$ and $\phi_{2}(x)$ at $x^{t}$, then $\varphi_{1}\left(x \mid x^{t}\right)+\varphi_{2}\left(x \mid x^{t}\right)$ is a surrogate function of $\phi_{1}(x)+\phi_{2}(x)$ at $x^{t}$.

We will give a whole derivation idea: our object is to create the surrogate function which is easy to minimize. As following, we will replace the minimization of the original cost function by minimizing at each iteration the surrogate function to obtain a new update. Moreover, by the definition above, the minimization of surrogate ensures the decrease of the original cost function.

Regardless of linear and non-linear SVM, let $B=A^{\prime} D$ or $K\left(A, A^{\prime}\right)^{\prime} D$ and $C=e^{\prime} D$,we simply the notion as:

$$
\begin{aligned}
& \left.\min F(u)=\frac{1}{2} u^{\prime}\left[\frac{I}{\beta}+B B^{\prime}+C C^{\prime}\right)\right] u-e^{\prime} u \\
& \text { s.t. } u \geq 0
\end{aligned}
$$

Then we can construct the surrogate for $F(u)$.

The key parts are to construct the surrogates for the following function.

$$
\begin{aligned}
& F_{1}(u)=\frac{1}{2} u^{\prime} B^{\prime} B u \\
& F_{2}(u)=\frac{1}{2} u^{\prime} B^{\prime} B u
\end{aligned}
$$

As can be seen, they have a similar form, so that we only give a detailed derivation for the first one.

\section{A. Surrogate for $F_{1}(u)$}

We can solve this optimization problem by the proposed method. Let $\mathrm{B}=\mathrm{B}^{+}-\mathrm{B}^{-}$, where $\mathrm{B}^{+}$and $\mathrm{B}^{-}$are two non-negative matrices, then

$$
f_{\text {mid }}\left(u \mid u^{t}\right)=\bar{f}\left(u \mid u^{t}\right)+\hat{f}\left(u \mid u^{t}\right)
$$

Where: 


$$
\begin{aligned}
& \bar{f}_{1}\left(u \mid u^{t}\right)=\frac{1}{4}\left\|2 B^{+} u-B^{+} u^{t}-B^{-} u^{t}\right\|^{2} \\
& \hat{f}_{1}\left(u \mid u^{t}\right)=\frac{1}{4}\left\|2 B^{-} u-B^{+} u^{t}-B^{-} u^{t}\right\|^{2}
\end{aligned}
$$

It can be verified that $f_{\text {mid }}\left(u \mid u^{t}\right)=F\left(u^{t}\right)$. By the convexity of $F(X)$, we view the two coefficients with value $1 / 2$ as the combination coefficients. It then shows that

$$
\begin{aligned}
& f_{\text {mid }}\left(X \mid X^{t}\right)=\frac{1}{4}\left\|2 B^{+} u-B^{+} u^{t}-B^{-} u^{t}\right\|^{2} \\
& +\frac{1}{4}\left\|-2 B^{-} u+B^{+} u^{t}+B^{-} u^{t}\right\|^{2} \geq G(X)
\end{aligned}
$$

We respectively construct surrogates for $\bar{f}\left(u \mid X^{t}\right)$ and $\hat{f}\left(X \mid X^{t}\right)$.Let

$$
\lambda_{1 j}^{+}=B_{i j}^{+} u_{j}^{t} /\left(B^{+} u^{t}\right)_{i}, \lambda_{1 i j}^{-}=B_{i j}^{-} u_{j}^{t} /\left(B^{-} u^{t}\right)_{i}
$$

then we obtain the surrogate as following:

$$
\begin{aligned}
& \overline{\bar{f}}_{1}\left(u \mid u^{t}\right)=\frac{1}{4} \sum_{i=1}^{M} \sum_{j=1}^{N} \lambda_{i j}^{+}\left[2 \frac{B_{i j}^{+} u_{j}}{\lambda_{l i j}^{+}}\right. \\
& \left.-\left(B^{+} u^{t}+B^{-} u^{t}\right)_{i}\right]^{2} \\
& \hat{\hat{f}}_{1}\left(u \mid u^{t}\right)=\frac{1}{4} \sum_{i=1}^{M} \sum_{j=1}^{N} \lambda_{i j}^{-}\left[2 \frac{B_{i j}^{-} u_{j}}{\lambda_{l i j}^{-}}\right. \\
& \left.-\left(B^{+} u^{t}+B^{-} u^{t}\right)_{i}\right]^{2}
\end{aligned}
$$

Take the first one as an example, it can be verified that $\overline{\bar{f}}_{1}\left(u^{t} \mid u^{t}\right)=\bar{f}_{1}\left(u^{t} \mid u^{t}\right)$ If considering Jensen's inequality and the convex combination coefficients $\lambda_{1 i j}^{+}=B_{i j}^{+} u_{j}^{t} /\left(B^{+} u^{t}\right)_{i},\left(\lambda_{1 i j}^{+} \geq 0\right.$ and $\left.\sum_{\mathrm{j}=1}^{\mathrm{N}} \lambda_{1 i j}^{+}=1\right)$,

then $\overline{\bar{f}}_{1}\left(u \mid u^{t}\right) \geq \bar{f}_{1}\left(u \mid u^{t}\right)$ is proven by the following inequality:

$$
\begin{aligned}
& \sum_{\mathrm{j}=1}^{\mathrm{N}} \lambda_{1 i j}^{+}\left[2 \frac{B_{i j}^{+} u_{j}}{\lambda_{1 i j}^{+}}-\left(B^{+} u^{t}+B^{-} u^{t}\right)_{i}\right]^{2} \\
& \geq\left[2 \sum_{j=1}^{N} B_{i j}^{+} u_{j}-\left(B^{+} u^{t}+B^{-} u^{t}\right)_{i}\right]^{2} \\
& \geq\left[2\left(B_{i j}^{+} u\right)_{i}-\left(B^{+} u^{t}+B^{-} u^{t}\right)_{i}\right]^{2}
\end{aligned}
$$

Thus we conclude that the surrogate of $F_{1}(u)$ is

$$
f_{1}\left(u \mid u^{t}\right)=\overline{\bar{f}_{1}}\left(u \mid u^{t}\right)+\hat{\hat{f}}_{1}\left(u \mid u^{t}\right)
$$

As can be seen, the variables of $f_{1}\left(u \mid u^{t}\right)$ are separable, so that its minimization is equivalent to some independent optimization problems of quadratic function with one unknown variable. So they are very easy to solve by a simple formula.

B. Surrogate for $F_{2}(u)$

We can construct the surrogate by the similar method. Let $C=C^{+}-C^{-}$, where $\mathrm{C}^{+}$and $\mathrm{C}^{-}$are two non-negative row vectors, and let

$$
\lambda_{2 j}^{+}=C_{j}^{+} u_{j}^{t} /\left(C^{+} u^{t}\right), \lambda_{2 j}^{-}=C_{j}^{-} u_{j}^{t} /\left(C^{-} u^{t}\right)
$$

then we obtain the surrogate for $\mathrm{F}_{2}(\mathrm{u})$. Note that we ignore the subscript $i$ because $C=e^{\prime} D$ is a row vector, which has no effect on the derivation process.

$$
f_{2}\left(u \mid u^{t}\right)=\overline{\bar{f}}_{2}\left(u \mid u^{t}\right)+\hat{\hat{f}}_{2}\left(u \mid u^{t}\right)
$$

Where

$$
\begin{gathered}
\overline{\bar{f}}_{2}\left(u \mid u^{t}\right)=\frac{1}{4} \sum_{j=1}^{N} \lambda_{2 j}^{+}\left[2 \frac{C_{j}^{+} u_{j}}{\lambda_{2 j}^{+}}-\left(C^{+} u^{t}+C^{-} u^{t}\right)\right]^{2} \\
\hat{\hat{f}}_{2}\left(u \mid u^{t}\right)=\frac{1}{4} \sum_{j=1}^{N} \lambda_{2 j}^{-}\left[2 \frac{C_{j}^{-} u_{j}}{\lambda_{2 j}^{-}}\right. \\
\left.-\left(C^{+} u^{t}+C^{-} u^{t}\right)\right]^{2}
\end{gathered}
$$

C. Derivation of Update rule

Let

$$
f\left(u \mid u^{t}\right)=\frac{u^{\prime} u}{2 \beta}+f_{1}\left(u \mid u^{t}\right)+f_{2}\left(u \mid u^{t}\right)-e^{\prime} u
$$

then it is the surrogate of $F(u)$. 
We can minimize it by taking the partial derivative and set to zero:

$$
\frac{\partial f\left(u \mid u^{t}\right)}{\partial u_{j}}=\frac{u_{j}}{\beta}+\frac{\partial f_{1}\left(u \mid u^{t}\right)}{\partial u_{j}}+\frac{\partial f_{2}\left(u \mid u^{t}\right)}{\partial u_{j}}-
$$

Where

$$
\begin{aligned}
\frac{\partial f_{1}\left(u \mid u^{t}\right)}{\partial u_{j}}= & 2 \frac{u_{j}}{u_{j}^{t}}\left\{\left[\left(B^{+}\right)^{\prime} B^{+}+\left(B^{-}\right)^{\prime} B^{-}\right] u^{t}\right\}_{j} \\
& -\left[\left(B^{+}+B^{-}\right)^{\prime}\left(B^{+}+B^{-}\right) u^{t}\right]_{j} \\
\frac{\partial f_{2}\left(u \mid u^{t}\right)}{\partial u_{j}}= & 2 \frac{u_{j}}{u_{j}^{t}}\left\{\left[\left(C^{+}\right)^{\prime} C^{+}+\left(C^{-}\right)^{\prime} C^{-}\right] u^{t}\right\}_{j} \\
& -\left[\left(C^{+}+C^{-}\right)^{\prime}\left(C^{+}+C^{-}\right) u^{t}\right]_{j}
\end{aligned}
$$

The partial derivative $\partial f\left(u \mid u^{t}\right) / \partial u_{j}=0$ is a linear equation with one unknown variable, which is easy to solve. Then we obtain the following updating algorithm:

$$
u_{j}^{t+1}=u_{j}^{t} \frac{1+\left(L_{1} u^{t}\right)_{j}+\left(L_{2} u^{t}\right)_{j}}{u_{j}^{t} / \beta+2\left(L_{3} u^{t}\right)_{j}+2\left(L_{4} u^{t}\right)_{j}}
$$

Where:

$$
\begin{aligned}
& L_{1}=\left(B^{+}+B^{-}\right)^{\prime}\left(B^{+}+B^{-}\right) \\
& L_{2}=\left(B^{+}\right)^{\prime} B^{+}+\left(B^{-}\right)^{\prime} B^{-} \\
& L_{3}=\left(C^{+}+C^{-}\right)^{\prime}\left(C^{+}+C^{-}\right) \\
& L_{4}=\left(C^{+}\right)^{\prime} C^{+}+\left(C^{-}\right)^{\prime} C^{-}
\end{aligned}
$$

If considering $C=e^{\prime} D$, where e is a column vector consisting of $1 \mathrm{~s}$ and $\mathrm{D}$ is a diagonal matrix consisting of 1 or $-1, \quad$ and denoting $C^{+}=\max (0, C) \quad$ and $C^{-}=-\min (0, C)$,then

$$
\begin{aligned}
& \left(L_{2} u^{t}\right)_{j}=\left[\left(C^{+}+C^{-}\right)^{\prime}\left(C^{+}+C^{-}\right) u^{t}\right]_{j}=e^{\prime} u^{t} \\
& \left(L_{4} u^{t}\right)_{j}=\left\{\left[\left(C^{+}\right)^{\prime} C^{+}+\left(C^{-}\right)^{\prime} C^{-}\right] u^{t}\right\}_{j}=e^{\prime} u^{t}
\end{aligned}
$$

we obtain a simple special example:

$$
u_{j}^{t+1}=u_{j}^{t} \frac{1+\left(L_{1} u^{t}\right)_{j}+\left(e^{\prime} u^{t}\right)_{j}}{u_{j}^{t} / \beta+2\left(L_{3} u^{t}\right)_{j}+2\left(e^{\prime} u^{t}\right)_{j}}
$$

The formula is very flexible and easy to implement, which we will give the MATLAB code for it.

Matlab code for (25):

function $u=\operatorname{SVM}(A, D$, Beta, IterNum)

[RowNum, ] = size $(\mathrm{A})$;

$\mathrm{u}=\operatorname{ones}($ RowNum, 1);

$\mathrm{B}=\mathrm{A} * * \mathrm{D}$

$\mathrm{PB}=\max (0, \mathrm{~B})$;

$\mathrm{NB}=-\min (0, \mathrm{~B})$;

$\mathrm{AB}=\operatorname{abs}(\mathrm{B})$

for $\mathrm{i}=1:$ IterNum;

$$
\begin{aligned}
& \mathrm{t}=\operatorname{sum}(\mathrm{u}) ; \\
& \mathrm{N}=1+\mathrm{AB}^{\prime} *\left(\mathrm{AB}^{*} \mathrm{u}\right)+\mathrm{t} ; \\
& \mathrm{D}=\mathrm{u} / \mathrm{Beta}^{\prime} 2^{*} \mathrm{~PB}^{\prime}\left(\mathrm{PB}^{*} \mathrm{u}\right) ; \\
& \mathrm{D}=\mathrm{D}+2 * \mathrm{NB}^{\prime} *\left(\mathrm{NB}^{*} \mathrm{u}\right)+2 * \mathrm{t} ; \\
& \mathrm{u}=\mathrm{u} . * \mathrm{~N} . / \mathrm{D} ;
\end{aligned}
$$

end

\section{Return}

In the next section, any decomposition of $\mathrm{B}$ and $\mathrm{C}$ will not have an effect on the result of the proposed. The update rule is flexible and easy to implement since it results from the current image multiplied by a factor. The update rule has two important properties: 1) the iterations are non-negative if the initial estimate satisfies $u_{j}^{0} \geq 0$ for any $\mathrm{j}$, and 2) the cost function monotonically decreases with increasing iteration number.

When we analyze the derivation process, we can make the following observation: the key step is to replace the minimization of $\mathrm{F}(\mathrm{u})$ by minimizing at each iteration the surrogate function $f\left(u \mid u^{t}\right)$ whose variables are separable. Moreover, $f\left(u \mid u^{t}\right) \geq F(u)$ and $f\left(u^{t} \mid u^{t}\right)=F\left(u^{t}\right)$; thus, the minimization of $f\left(u \mid u^{t}\right)$ ensures the decrease of $F(X)$.

\section{CONVERGENCE}

We will prove that the sequence $\left\{u^{t}\right\}$ converges to a global minimum. In theory, a global solution must meet the Kuhn-Tucker (KT) conditions, and vice versa if the cost function is convex. It is easy to see the strict convexity of $\mathrm{F}(\mathrm{u})$. By Theorem 2.19, the KT conditions are as follows:

$$
\begin{array}{lll}
\frac{\partial F(u)}{\partial u_{j}}=0 & \text { if } & \mathrm{u}_{\mathrm{j}} \succ 0 \\
\frac{\partial F(u)}{\partial u_{j}}=0 & \text { if } & \mathrm{u}_{\mathrm{j}}=0
\end{array}
$$


We entertain a important and reasonable assumptions.

Assumption 1: For the iteration sequence $\left\{X^{t}\right\}$, we assume that the algorithm starts from a positive image.

The assumption forces the iterations to be positive, but the limit may be zero. Now, We begin to prove the global convergence. First, we give several useful lemmas.

Lemma 1:The set of accumulation points of a bounded sequence $\left\{Z^{t}\right\}$ with $\left\{\left\|Z^{t+1}-Z^{t}\right\|\right\} \rightarrow 0$ is connected and compact.

Proof: This is Theorem 28.1 from Ostrowski. The reader is kindly referred to this paper for the proof.

Lemma 2: The iteration sequence $\left\{u^{t}\right\}$ is bounded.

Proof: We assume that $\mathrm{u}^{*}$ is the sole minimum, then expand $\mathrm{F}$ on $\mathrm{u}^{*}$ by Taylor series.

$$
\begin{aligned}
& F(u)-F\left(u^{*}\right) \\
& =\frac{1}{4}\left(u-u^{*}\right)^{T}\left[\frac{I}{\beta}+D\left(A A^{\prime}+e e^{\prime}\right) D\right]\left(u-u^{*}\right) \\
& \geq\left(u-u^{*}\right)^{\prime}\left(u-u^{*}\right) /(4 \beta)
\end{aligned}
$$

Let $L_{0}=F\left(u^{0}\right)-F\left(u^{*}\right)$, then $\left\{u^{t}\right\} \subset\left\{u:\left(u-u^{*}\right)^{\prime}\left(u-u^{*}\right) \leq \beta L_{0}\right\}$,

because $\left\{F\left(u^{t}\right)\right\}$ monotonously decreases, so $\left\{u^{t}\right\}$ is bounded.

Lemma 3: The sequence.

Proof: Take into account that

$$
\begin{aligned}
& \nabla_{j j}^{2} f\left(u \mid u^{t}\right)=\frac{1}{\beta}+2 \frac{\left\{\left[\left(B^{+}\right)^{\prime} B^{+}+\left(B^{-}\right)^{\prime} B^{-}\right] u^{t}\right\}_{j}}{u_{j}^{t}} \\
& +2 \frac{\left\{\left[\left(C^{+}\right)^{\prime} C^{+}+\left(C^{-}\right)^{\prime} C^{-}\right] u^{t}\right\}_{j}}{u_{j}^{t}} \geq \frac{1}{\beta}
\end{aligned}
$$

Since $\nabla f\left(u^{t+1} \mid u^{t}\right)=0$, , then

$$
\begin{aligned}
& F\left(u^{t}\right)-F\left(u^{t+1}\right) \geq f\left(u^{t} \mid u^{t}\right)-f\left(u^{t+1} \mid u^{t}\right) \\
& =\frac{1}{2}\left(u^{t}-X^{t+1}\right)^{\prime} \nabla^{2} f\left(u^{t+1} \mid u^{t}\right)\left(u^{t}-u^{t+1}\right) \\
& \geq \frac{1}{2 \beta}\left\|X^{t}-X^{t+1}\right\|^{2}
\end{aligned}
$$

Because $\left\{F\left(u^{t}\right)\right\}$ monotonically decreases and it is boundedfrom below, then $\left\{F\left(u^{t}\right)-F\left(u^{t+1}\right)\right\} \rightarrow 0$, so $\left\{\left\|u^{t}-u^{t+1}\right\|\right\} \rightarrow 0$.

Lemma 4: If a subsequence $\left\{u^{t_{s}}\right\} \rightarrow u^{*}$, then $\left\{u^{t_{s}+1}\right\} \rightarrow u^{*}$ as well.

Proof: By contradiction, if $\left\{u^{t_{s}+1}\right\}$ diverges, then it must have a convergent subsequence $\left\{u^{t_{s_{s}}+1}\right\} \rightarrow u^{* *} \neq u^{*}$ because of the boundness by Lemma 2 . Let $\varepsilon_{0}=\left\|u^{*}-u^{* *}\right\| \succ 0$, and we consider the two convergent sub-sequences $\left\{u^{t_{s_{s}}}\right\}$ and $\left\{u^{t_{s_{s}}+1}\right\}$, then there must be a positive integer $\mathrm{T}$ to make $\left\{\left\|u^{t_{s_{s}}}-u^{*}\right\|\right\} \prec \varepsilon_{0} / 4$ when $t_{s_{s}} \succ T$. By the triangle inequality, we can obtain the contradictive result to $\left\{\left\|u^{t}-u^{t+1}\right\|\right\} \rightarrow 0$ as

$$
\begin{aligned}
& \mid u^{t_{s_{s}}}-u^{t_{s_{s}}+1}\|+\| u^{t_{s_{s}}}-u^{*}\|+\| u^{t_{s_{s}}+1}-u^{* *} \| \\
& \geq\left\|u^{*}-u^{* *}\right\| \Rightarrow\left\|u^{t_{s_{s}}}-u^{t_{s_{s}}+1}\right\| \succ \varepsilon_{0} / 2
\end{aligned}
$$

Lemma 5: At each iteration, we know that

$$
\begin{aligned}
& u_{j}^{t+1}=u_{j}^{t}-\alpha_{j}\left[\nabla F\left(u^{t}\right)\right]_{j} \\
& \alpha_{j}= \\
& \frac{u_{j}^{t}}{u_{j}^{t} / \beta+2\left\{\left[\left(B^{+}\right)^{\prime} B^{+}+\left(B^{-}\right)^{\prime} B^{-}\right] u^{t}\right\}_{j}+2\left(e^{ \pm}\right)^{\prime} u^{t}}
\end{aligned}
$$

Proof: We consider that

$$
\begin{aligned}
\frac{\partial f_{1}\left(u^{t} \mid u^{t}\right)}{\partial u_{j}}= & 2\left\{\left[\left(B^{+}\right)^{\prime} B^{+}+\left(B^{-}\right)^{\prime} B^{-}\right] u^{t}\right\}_{j} \\
& -\left[\left(B^{+}+B^{-}\right)^{\prime}\left(B^{+}+B^{-}\right) u^{t}\right]_{j} \\
= & 2\left[\left(B^{+}-B^{-}\right) u^{t}\right]_{j}=\frac{\partial F_{1}\left(u^{t}\right)}{\partial u_{j}} \\
\frac{\partial f_{2}\left(u \mid u^{t}\right)}{\partial u_{j}}= & 2 \frac{u_{j}}{u_{j}^{t}}\left\{\left[\left(C^{+}\right)^{\prime} C^{+}+\left(C^{-}\right)^{\prime} C^{-}\right] u^{t}\right\}_{j} \\
& -\left[\left(C^{+}+C^{-}\right)^{\prime}\left(C^{+}+C^{-}\right) u^{t}\right]_{j} \\
= & 2\left[\left(C^{+}-C^{-}\right) u^{t}\right]_{j}=\frac{\partial F_{2}\left(u^{t}\right)}{\partial u_{j}}
\end{aligned}
$$


It is easy to verify the correctness of (32) if replacing $\nabla F_{2}\left(u^{t}\right)$ and $\nabla F_{2}\left(u^{t}\right)$ by (33) and (34). By the three theorems below, the global convergence will be proven.

Theorem 1: Let $\left\{u^{t_{s}}\right\} \rightarrow u^{*}$ be any convergent subsequence, then $\mathrm{u}^{*}$ meets the first KT condition (26).

Proof: When $u_{j}^{*} \succ 0$, it is easy to obtain that $\left[\nabla F\left(u^{*}\right)\right]_{j}=\left[\nabla f\left(u^{*} \mid u^{*}\right)\right]_{j} \quad$. $\quad$ In addition $\partial F\left(u \mid u^{t_{s}}\right) /\left.\partial u_{j}\right|_{u^{t_{s}+1}}=0$, and $\left\{u^{t_{s}}\right\} \rightarrow u^{*} \quad$,so that $\left\{u^{t_{s}+1}\right\} \rightarrow u^{*}($ Lemma 4$)$, then

$$
\left.\frac{\partial F\left(u \mid u^{*}\right)}{\partial u_{j}}\right|_{u^{*}}=\left.\lim \frac{\partial F\left(u \mid u_{s}^{t}\right)}{\partial u_{j}}\right|_{u^{t_{s}}+1}=0
$$

Theorem 2: The whole sequence $\left\{u^{t}\right\}$ converges.

Proof: According to Lemma 1, 2 and 3, the set of accumulation points of $\left\{u^{t}\right\}$ is connected and compact. If we can prove that the number of accumulation points is finite, then the desired result follows because a finite set can be connected only if it consists of a single point

To prove the existence of a finite number of accumulation points, we consider any accumulation point . Given an integer set $\Omega=\{1,2, \ldots, \mathrm{T}\}$ where $\mathrm{T}$ is the total number of components of $\mathrm{u}$, then $\Omega^{*}=\left\{j: u_{j}^{*}=0\right\}$ is a subset of $\Omega$. Let $F_{\Omega^{*}}$ be the restrictions of $\mathrm{F}$ to the set $\left\{u: u_{j}=0, j \in \Omega^{*}\right\}$, which is a strictly convex function of the reduced variables. It follows that $F_{\Omega^{*}}$ has a unique minimum (Theorem 1: $\partial f\left(u^{*}\right) / \partial u_{j}=0$ if $u_{j}^{*} \succ 0$ ). It means that an accumulation point must correspond to a subset of $\Omega$. The number of subsets of $\Omega$ is finite, so the number of accumulation points is also finite. In Theorem 1, we prove that every accumulation point meets the first KT condition, by which the full sequence convergence is provided in Theorem 2. Naturally, the limit of $\left\{X^{t}\right\}$ satisfies the first KT condition. As below, we will show that the second KT condition is satisfied.

Theorem 3: The limit $\mathrm{X}^{*}$ of $\left\{u^{t}\right\}$ meets the second KT condition (27).

Proof: When $u_{j}^{*}=0$, by contradiction, we assume that there is an $u_{j}^{*}=0$ satisfying $\left[\nabla F\left(u^{*}\right)\right]_{j} \prec 0$. Since $\left\{u^{t}\right\} \rightarrow X^{*}$, there exists an $\varepsilon \prec 0$ and a positive integer $\mathrm{T}$ such that $\left[\nabla F\left(u^{*}\right)\right]_{j} \prec \varepsilon$ for $\mathrm{t}>\mathrm{T}$, then

$$
u_{j}^{t+1}-u_{j}^{t}=-\alpha_{j}\left[\nabla F\left(u^{*}\right)\right]_{j} \succ-\alpha_{j} \varepsilon \succ 0
$$

\section{(by Lemma 5)}

Thus, we can obtain that $u_{j}^{t+1} \succ u_{j}^{t}$, which is contradicted to $\quad\left\{u_{j}^{t}\right\} \rightarrow 0$.

\section{SIMULATION}

The experiments were performed on HP Compaq PC with3.00 GHz Core i5 CPU and 4 GB memory. The algorithms were implemented in MATLAB 7.0. All of the algorithms were initiated by the same vector e for a fair comparison.

\section{A. Verification Testing}

First of all, two classical SVM algorithms lib-SVM and $\mathrm{SVM}^{\text {light }}$ were chosen as comparison. Then different data sets were used to verify the reliability of the new algorithm. Finally, in biomedical application, we analyzed the data set of diabetes in 130-US hospitals for years 1999-2008. The data set represents 10 years (1999-2008) of clinical care at 130 US hospitals and it includes over 50 features representing patient.

Experiment 1: The size of data sets in the experiment differs from 4192 to 581012 . When working on the same data set, the time and accuracy of classification are different by the three algorithms.

The result in figure 2(a) shows the classification accuracy of three algorithms on different data sets. When training data sets, we choose the loss factor (from $2^{-10}$ to $2^{10}$ ), corresponding to the different classification accuracy. With the data size increasing, the precision is different. LibSVM and SBM are similar, the classification rate of $\mathrm{SVM}^{\text {light }}$ is lower.

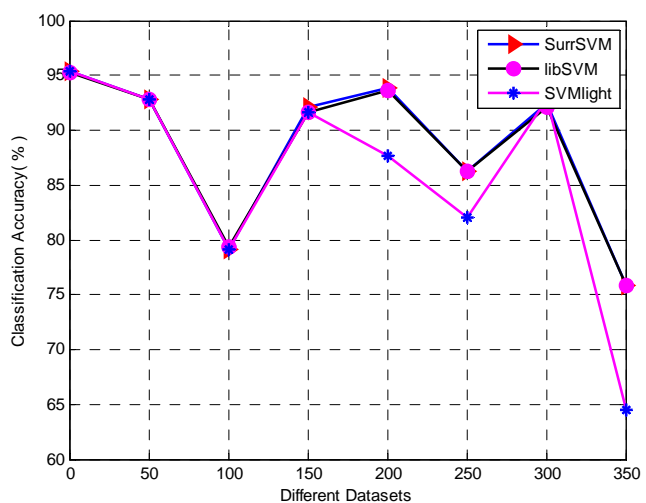

FIGURE II. (A). THE CLASSIFICATION ACCURACY OF TEST ONE 


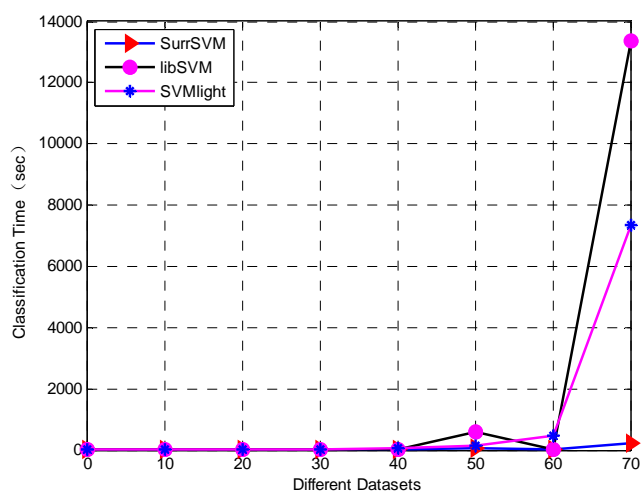

FIGURE II. (B). THE CLASSIFICATION TIME OF TEST ONE

Graphical interpretation in Figure 2(b) shows the classification time of different data sets in these three algorithms. When the size increases, SBM classifies the data faster significantly.

experiment 2:On the basis of the experimental one, we select NDC(Normally Distributed Clusters) data generator to get some sets of NDC data to study the influence of data dimension on the classification efficiency. The experimental results are shown below:

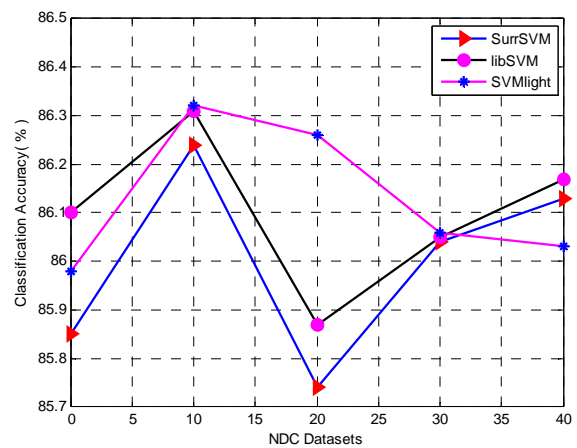

FIGURE III. (A). THE CLASSIFICATION ACCURACY OF TEST TWO

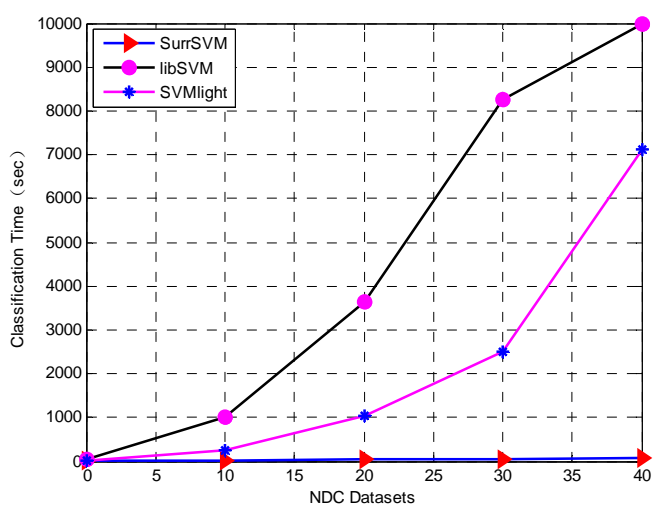

FIGURE III. (B). THE CLASSIFICATION TIME OF TEST TWO

Figure 3. Graphical interpretation shows for the same data set, when data dimension increases(the size of data sets from left to right are respectively 20 thousands, 100 thousands, 200 thousands, 300 thousands and 500 thousands),SBM could keep classification accuracy steady and reduce the classification time.

experiment 3:In order to study the impact of data dispersion degree to the classification efficiency, we made the third proof test. By adjusting parts of the data generator parameters, data sets with different discrete degree were obtained. As the name implies, the greater discrete degree, the more scattered data. The experimental results are shown below:

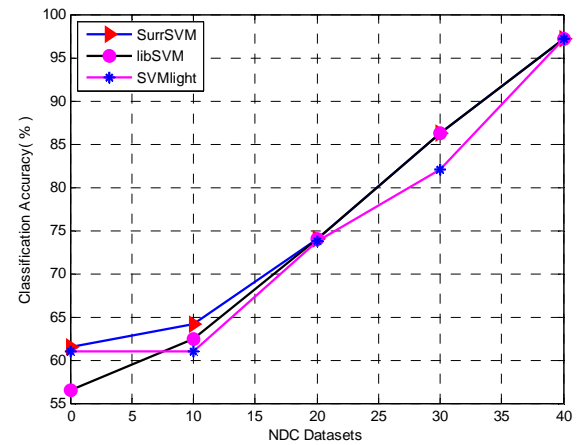

FIGURE IV. (A). THE CLASSIFICATION ACCURACY OF TEST THREE

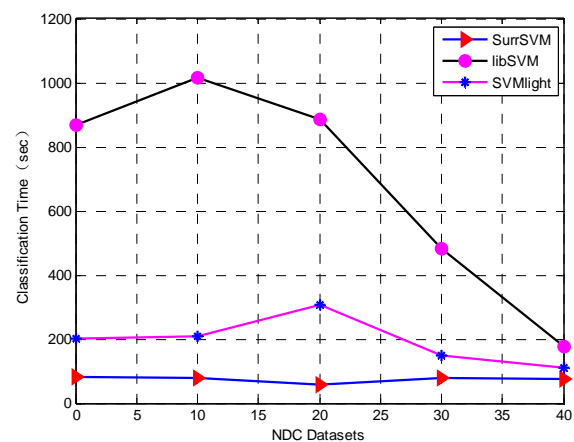

FIGURE IV. (B). THE CLASSIFICATION TIME OF TEST THREE

Figure 4. Graphical interpretation shows that the classification accuracy in three algorithms are similar but the time for classification has a large difference. Compared with other two methods showed a dramaticlly advantage.

\section{B. Application Testing}

By Verification testing, we can see that SBM has a better performance in large-scale data classification. This section is mainly to research in the field of practical application. The data we use is 10 years (1999-2008) of clinical care at 130 US hospitals, which includes over 50 features representing patient and hospital outcomes. After data processing, we kept the information that reflect patients characteristics. Then we use libSVM, SVM ${ }^{\text {light }}$ and SBM to classify the processed data and obtain the following result: 
TABLE I. THE CLASSIFICATION ACCURACY OF THE DATA SET

\begin{tabular}{|c|c|c|c|}
\hline $\begin{array}{c}\text { Algorithm } \\
\text { Data se` }\end{array}$ & SBM & libSVM & SVMlight \\
\hline $\begin{array}{c}\text { Accuracy } \\
\text { (Persent) }\end{array}$ & 99.98 & 99.92 & 99.87 \\
\hline
\end{tabular}

TABLE II. THE CLASSIFICATION TIME OF THE DATA SET

\begin{tabular}{|c|c|c|c|}
\hline $\begin{array}{c}\text { Algorithm } \\
\text { Data set }\end{array}$ & SBM & libSVM & SVMlight \\
\hline $\begin{array}{c}\text { Time } \\
\text { (Seconds) }\end{array}$ & 36.43 & 518.35 & 273.90 \\
\hline
\end{tabular}

From the results above,we can get that the new algorithm shows a high classification efficiency in the practical application.

\section{V.CONCLUSION}

This paper mainly introduces a new kind of SVM algorithm:Surrogate-based SVM method (SBM).By theoretical derivation, we find that SBM has many favorable properties and it is easy to parallelize. We choose classical SVM algorithms lib-SVM and SVM ${ }^{\text {light }}$ as comparison. After simulation of different perspectives, the algorithm we proposed showed a strong advantage in large-scale classification. SBM not only makes classification accuracy stable, but also greatly reduces the classification time.

\section{ACKNOWLEDGEMENTS}

Lichman, M. (2013). UCI Machine Learning Repository [http://archive.ics.uci.edu/ml]. Irvine, CA: University of California, School of Information and Computer Science.

The authors gratefully acknowledge Beata Strack, Jonathan P. DeShazo, Chris Gennings, Juan L. Olmo, Sebastian Ventura, Krzysztof J. Cios, and John N. Clore, "Impact of HbAlc Measurement on Hospital Readmission Rates: Analysis of 70,000 Clinical Database Patient Records." BioMed Research International, vol. 2014, Article ID 781670, 11 pages, 2014.

This work was supported by the National Natural Science Foundations of China (61302013, 61372014), Science and Technology Plan of Liaoning Province of China (2014305001) and Fundamental Research Funds for the Central Universities of China( N141008001).

\section{REFERENCES}

[1] D.D. Lee, and H.S. Seung, "Learning the parts of objects by non-negative matrix factorization," Nature, vol. 401, pp. 788-791, 1999.

[2] D.D. Lee, and H.S. Seung, "Algorithms for non-negative matrix factorization," in Adv. Neural Inform. Processing, USA, pp. 556-562, 2000 .

[3] I.S. Dhillon, and S. Sra, "Generalized nonnegative matrix approximations with bregman divergences," in Proc. Neural Inform. Processing Syst., USA, pp. 283-290, 2005.

[4] A. Cichocki, R. Zdunek, and S. Amari, "Csiszar's divergences for non-negative matrix factorization: family of new algorithms," Lect. Notes Comput. Sci., vol. 3889, pp. 32-39, 2006.

[5] A. Cichockia, H. Lee, Y.D. Kimb, and S.J. Choi, "Non-negative matrix factorization with $\alpha$-divergence," Pattern Recogn. Lett., vol. 29, pp. 1433-1440, 2008.
[6] C. Fevotte, N. Bertin, and J. Durrieu, "Nonnegative matrix factorization with the itakurasaito divergence: with application to music analysis," Neural Computat., vol. 2, pp. 793-830, 2009.

[7] A. Cichocki, and S. Amari, "Families of alpha- beta- and gamma-divergences: flexible and robust measures of similarities," Entropy, vol. 12, pp. 1532-1568, 2010.

[8] A. Cichocki, S. Amari, R. Zdunek, P. Kompass, G. Hori, and Z. $\mathrm{He}$, "Extended smart algorithms for non-negative matrix factorization," Lect. Notes Artif. Intel., vol. 4029, pp. 548-562, 2006.

[9] R. Zdunek, and A. Cichocki, "Gibbs regularized nonnegative matrix factorization for blind separation of locally smooth signals," in Proc. 15th IEEE Int. Workshop Nonlinear Dynamics Electronic Syst. (NDES07), Japan, pp. 317-320, 2007.

[10] C.J. Lin, "On the convergence of multiplicative update algorithms for nonnegative matrix factorization," IEEE Trans. Neural Networks, vol. 18, pp. 1589-1596, 2007.

[11] G.T. Herman, and D. Odhner, "Performance evaluation of an iterative image reconstruction algorithm for positron emission tomography," IEEE Trans. Med. Imag., vol. 10, pp. 336-346, 1991.

[12] P. Green, "Bayesian reconstruction for emission tomography data using a modified em algorithm," IEEE Trans. Med. Imag., vol. 9, pp. 84-93, 1990.

[13] W.I. Zangwill, Nonlinear Programming: A Unified Approach. New York, USA: Prentice-Hall, 1969.P.O. Hoyer, ”Non-negative matrix factorization with sparseness con-straints," J. Mach. Learn. Res., vol. 5 , pp. 1457-1469, 20 